\title{
Ambulantes Management nach vorzeitigem Blasensprung - eine chancenreiche Alternative auch für das geburtshilfliche Outcome
}

Anne-Katrin Klotzsch, Weijing Li, Monique Kaiser, Marie Hurpin, Michael Abou-Dakn

Das ambulante Management nach vorzeitigem Blasensprung am Termin erlaubt es, dass Frauen sich möglichst lang in ihrer vertrauten Umgebung aufhalten. Seit September 2017 wird das ambulante Procedere im St. Joseph Krankenhaus Berlin-Tempelhof erfolgreich praktiziert. Nun liegen die Ergebnisse einer retrospektiven, quantitativen Datenerhebung vor, die die positiven Effekte hinsichtlich des geburtshilflichen Outcomes bestätigen.

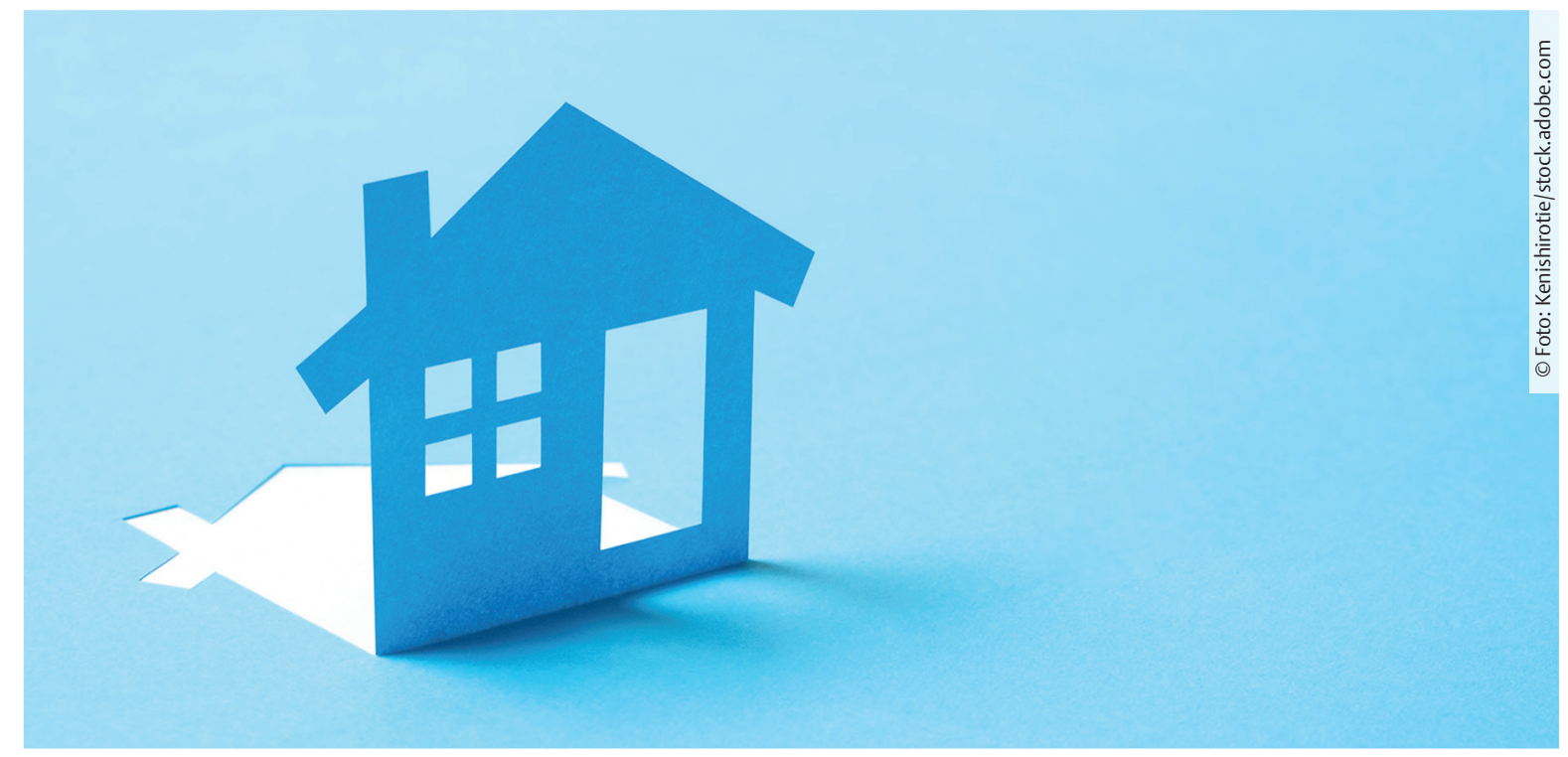

Abb.1 Durch das ambulante Management nach vorzeitigem Blasensprung am Termin, können sich Frauen länger in ihrem vertrauten häuslichen Umfeld aufhalten. (৫ Kenishirotie/stock.adobe.com; Symbolbild)

\section{Hintergrund}

Seit September 2017 wird im St. Joseph Krankenhaus Berlin-Tempelhof das ambulante Management nach vorzeitigem Blasensprung (VBS) am Termin erfolgreich praktiziert. Frauen ohne geburtshilfliches Risiko dürfen sich entscheiden, ob sie die erste Zeit nach diagnostiziertem Blasensprung in der Klinik oder in ihrem häuslichen Umfeld verbringen möchten.
In DIE HEBAMME 4/19 wurde über die Implementierung und die ersten Erfahrungen und Vorteile des ambulanten Managements berichtet. Mittlerweile wurden klinikinterne Daten statistisch ausgewertet, die bestätigen, dass ein ambulantes Management eine sicherere und gewinnbringende Alternative zur stationären Aufnahme darstellt. 


\section{Aktuell praktiziertes Vorgehen}

Inzwischen ist das Vorgehen bei allen involvierten Berufsgruppen routiniert und gehört zum regulären Behandlungsangebot für die zu betreuenden Frauen und Paare. Die Erstversion des neuen Standards wurde hinsichtlich der Einschlusskriterien und der Aufenthaltsdauer im häuslichen Umfeld erweitert.

Den Frauen wird es nun ermöglicht, sich bis zum Zeitpunkt 24 Stunden nach Blasensprung bzw. bis zum darauffolgenden Morgen (d.h. länger als 24 Stunden) zu Hause aufzuhalten, bevor ihnen zu einer stationären Aufnahme bzw. einer Einleitung geraten wird.

Eine Antibiose zur Infektionsprophylaxe wird nur bei klinischen Anzeichen, wie z.B. erhöhtem Infektlabor, maternalem Fieber $>38^{\circ} \mathrm{C}$ über 30 Minuten oder persistierender fetaler Tachykardie durchgeführt und bedarf einer vorzeitigen stationären Aufnahme.

\section{Aktuelle Datenlage}

Im Rahmen der Implementierung des ambulanten Managements wurden nationale und internationale Studien einbezogen, die das perinatale Outcome nach ambulantem Vorgehen nach VBS am Termin untersuchten [10].

Auch weiterhin zeigt die Literatur kontroverse Meinungen auf und ist zur Stärkung der Allgemeingültigkeit noch ausbaufähig. Während das ambulante Management in internationalen Leitlinien fest verankert ist [1][2] [3], gibt es in der deutschen Leitlinie der AWMF und DGGG hierzu keine Empfehlung. Diese befindet sich jedoch aktuell in Überarbeitung [4].

Inwieweit das ambulante Procedere in Deutschland bereits praktiziert wird, lässt sich nicht genau sagen. Im Rahmen einer Querschnittstudie wurde eine Online-Umfrage an 85 Geburtsinstitutionen im deutschsprachigen Raum geschaltet, worauf 47 Rückmeldungen eingingen. Aus den Antworten ging hervor, dass mehr als die Hälfte dieser Kliniken und Geburtshäuser ein ambulantes Management anbietet und die Mehrzahl dieser Institutionen über mehrjährige Erfahrung damit verfügt. Besonders die Zufriedenheit der Schwangeren wurde betont. Es konnten jedoch keine Erkenntnisse zu fetalen Parametern abgeleitet werden [5].

Eine Studie von Vetter et al. untersuchte 61 Patientinnen, die nach strengen Einschlusskriterien ambulant $(n=30)$ bzw. stationär $(n=31)$ betreut wurden. Die retrospektive Auswertung betrachtete maternale Faktoren (Einleitung, Geburtsmodus, Antibiotikabedarf) und fetale Faktoren ( $\mathrm{pH}$ arteriell, 5 min APGAR) sowie die Hospitalisation bis zur Geburt. Bis auf eine verkürzte
Tab.1 Kriterien für eine ambulante Betreuung

\begin{tabular}{|c|c|}
\hline Maternal & Fetal \\
\hline 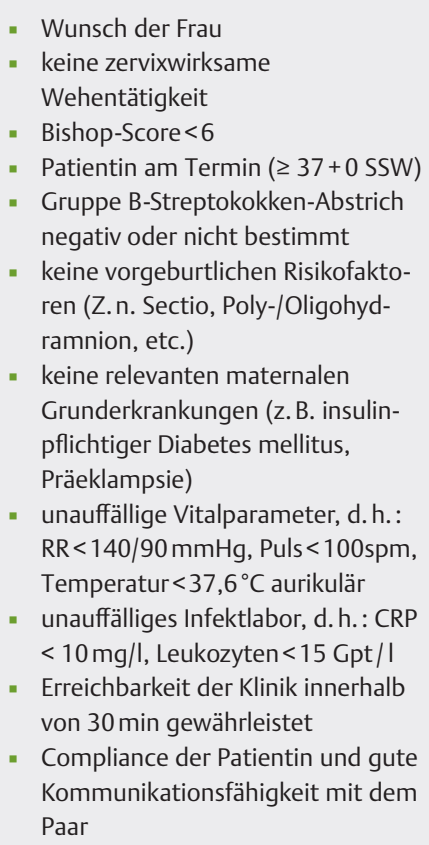 & $\begin{array}{l}\text { " Klares Fruchtwasser } \\
\text { " Schädellage; Kopf fest im } \\
\text { Becken } \\
\text { - CTG und Kindsbewegungen } \\
\text { unauffällig }\end{array}$ \\
\hline
\end{tabular}

Hospitalisationsdauer der ambulant geführten Patientinnengruppe, wurden keine signifikanten Unterschiede der zwei Gruppen festgestellt [6].

Erstvorstellung bei vorzeitigem Blasensprung am Termin $\downarrow$

CTG, vaginale Untersuchung und Verifizierung des VBS, Blutentnahme, Erhebung der Vitalzeichen und Prüfung der Einschlusskriterien nach Risikokatalog durch die Hebamme $\rightarrow$ Informationsgespräch durch die Hebamme

Aufklärungsgespräch und Entlassung der Frau nach Einsicht der Blutergebnisse in ihr häusliches Umfeld bis $12 \mathrm{~h}$ nach Blasensprung bzw. bis zum darauffolgenden Morgen durch die Ärztin/den Arzt

Einbestellung zum Kontrollbesuch in die Klinik

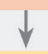

CTG und Vitalzeichenkontrolle durch die Hebamme $\rightarrow$ bei unauffälligem CTG darf die Frau in Rücksprache mit der Ärztin/dem Arzt bis zum Zeitpunkt $24 \mathrm{~h}$ nach VBS

bzw. bis zum darauffolgenden Morgen (d.h. länger als $24 \mathrm{~h}$ ) nach Hause geschickt werden.

Danach erfolgt die stationäre Aufnahme und - wenn notwendig die Empfehlung einer Einleitung

Abb. 2 Übersichtsgrafik des ambulanten Vorgehens (๔ A.-K. Klotzsch; edited by Thieme Group). 
Tab. 2

\begin{tabular}{|c|c|c|c|c|}
\hline Parameter & Gesamt ( $n=158)$ & Stationär $(n=87)$ & Ambulant $(n=71)$ & p-Wert \\
\hline \multicolumn{5}{|c|}{ Generelle Informationen zur Frau } \\
\hline Alter (Jahre, mean \pm SD) & $31.51 \pm 5.40$ & $32.16 \pm 5.95$ & $30.72 \pm 4.55$ & 0.277 \\
\hline Parität (0/1/2/3/9) & $119 / 33 / 4 / 1 / 1$ & $67 / 18 / 1 / 0 / 1$ & $52 / 15 / 3 / 1 / 0$ & 0.490 \\
\hline \multicolumn{5}{|c|}{ Parameter in Bezug auf den Geburtsprozess } \\
\hline $\begin{array}{l}\text { MM-Weite zur stationären } \\
\text { Aufnahme (mean } \pm \text { SD) }\end{array}$ & $1.33 \pm 1.43$ & $1.45 \pm 1.74$ & $1.20 \pm 0.92$ & 0.014 \\
\hline $\begin{array}{l}\text { Entwicklung spontaner } \\
\text { WTK (J/N/ns) }\end{array}$ & $108 / 50 / 0$ & $48 / 39 / 0$ & $60 / 11 / 0$ & 0.000 \\
\hline $\begin{array}{l}\text { Medizinische Geburtsein- } \\
\text { leitung (J/N/ns) }\end{array}$ & $59 / 99 / 0$ & $45 / 42 / 0$ & $14 / 57 / 0$ & 0.000 \\
\hline $\begin{array}{l}\text { Anzahl vag U von Diag- } \\
\text { nose VBS bis Zeitpunkt } \\
\text { Wehenbeginn (mean } \pm S D \text { ) }\end{array}$ & $1.56 \pm 0.83$ & $1.60 \pm 0.93$ & $1.52 \pm 0.67$ & 0.080 \\
\hline $\begin{array}{l}\text { Anzahl vag U von Diag- } \\
\text { nose VBS bis Zeitpunkt } \\
\text { Geburt (mean } \pm \text { SD) }\end{array}$ & $6.32 \pm 3.40$ & $6.48 \pm 3.32$ & $6.11 \pm 3.50$ & 0.381 \\
\hline $\begin{array}{l}\text { Zeitspanne von Zeitpunkt } \\
\text { BS bis Beginn WTK } \\
\text { (h, mean } \pm \text { SD) }\end{array}$ & $14.69 \pm 11.12$ & $18.08 \pm 12.49$ & $10.54 \pm 7.35$ & 0.001 \\
\hline $\begin{array}{l}\text { Zeitspanne von Zeitpunkt } \\
\text { BS bis zur Geburt } \\
\text { (h, mean } \pm \text { SD) }\end{array}$ & $23.81 \pm 13.92$ & $28.25 \pm 14.90$ & $18.37 \pm 10.35$ & 0.001 \\
\hline $\begin{array}{l}\text { Wehenaugmentation } \\
(\mathrm{J} / \mathrm{N} / \mathrm{ns})\end{array}$ & $72 / 86 / 0$ & $44 / 43 / 0$ & $28 / 43 / 0$ & 0.162 \\
\hline $\begin{array}{l}\text { Geburtsmodus (SP/VO/ } \\
\text { SS/NS/ns) }\end{array}$ & $102 / 38 / 16 / 0 / 2$ & $63 / 16 / 7 / 0 / 1$ & $39 / 22 / 9 / 0 / 1$ & 0.104 \\
\hline $\begin{array}{l}\text { Infektionszeichen Mutter } \\
\text { subpartal (J/N/ns) }\end{array}$ & $23 / 135 / 0$ & $14 / 73 / 0$ & $9 / 62 / 0$ & 0.545 \\
\hline $\begin{array}{l}\text { Analgesie allgemein } \\
(\mathrm{J} / \mathrm{N} / \mathrm{ns})\end{array}$ & $108 / 50 / 0$ & $57 / 30 / 0$ & $51 / 20 / 0$ & 0.396 \\
\hline $\mathrm{PDA}(\mathrm{J} / \mathrm{N} / \mathrm{ns})$ & $66 / 90 / 2$ & $35 / 50 / 2$ & $31 / 40 / 0$ & 0.754 \\
\hline $\begin{array}{l}\text { Anzahl Tage stationärer } \\
\text { Aufenthalt }(\mathrm{d} \text {, mean } \pm S D \text { ) }\end{array}$ & $4.29 \pm 1.26$ & $4.28 \pm 1.25$ & $4.30 \pm 1.29$ & 0.077 \\
\hline \multicolumn{5}{|l|}{ Neonatale Parameter } \\
\hline $\begin{array}{l}\text { Verlegung NG auf Neona- } \\
\text { tologie (J/N/ns) }\end{array}$ & $18 / 140 / 0$ & $12 / 75 / 0$ & $6 / 65 / 0$ & 0.293 \\
\hline $\begin{array}{l}\text { Infektion NG als Verle- } \\
\text { gungsgrund (J/N/ns) }\end{array}$ & $10 / 8 / 140$ & $8 / 4 / 75$ & $2 / 4 / 65$ & 0.300 \\
\hline
\end{tabular}

mean $\pm \mathrm{SD}=$ Durchschnitt \pm Standardabweichung, $\mathrm{J}=\mathrm{Ja}, \mathrm{N}=$ Nein, $\mathrm{ns}=$ nicht dokumentiert, $\mathrm{SP}=$ Spontanpartus, $\mathrm{VO}=$ vaginal-operative Entbindung, SS = sekundäre Sectio, NS = Notsectio

$p<0.01$ signifikant $\mathrm{p}<0.001$ hoch signifikant

Hinweis: Der p-Wert/p-value ist ein statistisches Maß bei einem Hypothesentest und misst die Wahrscheinlichkeit, ob der beobachtete Unterschied zwischen zwei Gruppen zufällig entstanden sein könnte. Je geringer der Wert ist, desto statistisch signifikanter ist der beobachtete Unterschied und somit die Wahrscheinlichkeit, dies auf die Grundgesamtheit zu übertragen [9].

In einer weiteren Untersuchung wurde eine größere Personengruppe inkludiert (stationär $=2020$, ambulant $=$ 37). Auch hier konnten keine statistischen Unterschiede bewiesen werden. Da die Daten durch die retrospektive Sichtweise schwach zu bewerten sind, wird ein individuelles Vorgehen empfohlen [7].
Das American College of Obstetricians and Gynecologists veröffentlichte 2019 ebenfalls eine retrospektive Studie, die 127 Frauen mit VBS am Termin ohne geburtshilfliches Risiko einschloss (stationär $=74$, ambulant $=53$ ). Die Zeitspanne vom Zeitpunkt des Blasensprungs bis zur Geburt war in der ambulanten Gruppe signifikant länger, 
die stationäre Aufenthaltsdauer jedoch kürzer. Es gab keinen signifikanten Unterschied in den sekundären Parametern, wie z.B. Verlegung in die Neonatologie, Geburtsmodi etc. [8].

\section{Retrospektive, quantitative Datenerhebung in Bezug auf das geburtshilfliche Outcome}

Durch ein interdisziplinäres Team des St. Joseph Krankenhauses Berlin-Tempelhof wurde eine klinikinterne, quantitative, retrospektive Datenerhebung durchgeführt ( $>$ Tab. 2). Zunächst wurden die Perinataldaten in der Klinikdatenbank ermittelt und danach mittels Akteneinsicht ausgelesen. Die Auswertung und Darstellung der Daten erfolgte mittels deskriptiver Statistik durch die Software IBM SPSS Statistics (T-test und Chi-Square-Test) und Microsoft Excel.

Hierbei wurden die ambulant geführten Frauen $(n=71)$ mit den stationären Frauen $(n=87)$ verglichen, die keine ambulante Betreuung wünschten. Beide Gruppen wiesen kein geburtshilfliches Risiko auf.

Die Datenerhebung bezog sich auf den Zeitraum vom 14.9.2017 bis 13.9.2018. Dies entspricht dem ersten Jahr nach Implementierung des ambulanten Procederes.

\section{Auswertung der Ergebnisse}

Es konnten signifikante Ergebnisse aus der Auswertung abgeleitet werden:

- Ambulant geführte Frauen hatten bei der stationären Aufnahme in den Kreißsaal einen durchschnittlich reiferen und günstigeren Muttermundsbefund.

- Die ambulante Gruppe entwickelte häufiger spontane Wehentätigkeit und brauchte weniger medizinische Geburtseinleitungen.

- Die Zeitspanne vom Zeitpunkt des Blasensprungs bis zum Beginn der Wehentätigkeit sowie bis zur Geburt war kürzer.

Aus den Ergebnissen kann geschlussfolgert werden, dass sich Frauen, die sich nach VBS in ihrem häuslichen und gewohnten Umfeld aufhalten, durch ihren entspannten Zustand besser auf die Geburt einlassen können. Somit kommt es eher zu einem natürlichen Wehenbeginn und adäquater Muttermundseröffnung vor Wiederaufnahme in die Klinik.

Interventionen hinsichtlich einleitender Maßnahmen sind weniger wahrscheinlich, genauso wie protrahierte Geburtsverläufe. Diese Umstände fördern die Physiologie der Geburt und Mutter-Kind-Bindung, was ein positives Geburtserleben begünstigt.

Die Erkenntnisse unterstützen eindeutig die These, dass das ambulante Management nach VBS am Termin bei
Frauen ohne geburtshilfliches Risiko Vorteile hinsichtlich des Outcomes aufweist und ein sicheres Procedere darstellt.

Jedoch sollten die klinikeigenen Erkenntnisse mit Bedacht betrachtet werden, da diese durchaus Limitierungen zeigen. Im Rahmen der Datenrecherche hat das interdisziplinäre Team festgestellt, dass die Dokumentation zum Teil lückenhaft war oder eine Diskrepanz zwischen den schriftlich verfassten und digital hinterlegten Daten vorlag. Es wird vermutet, dass dies auf eine erhöhte Arbeitsbelastung im klinischen Setting und einen Zeit- und Fachkräftemangel zurückzuführen ist. Des Weiteren ist die Vergleichsgruppe der stationären Frauen nur retrospektiv gefiltert und garantiert somit keinen exakten Wert, wohingegen die Gruppe der ambulanten Frauen im genannten Untersuchungszeitraum eindeutig nachvollziehbar war.

INFO

Neben der Untersuchung der geburtshilflichen Daten wurde das Feedback der Frauen zum Angebot des ambulanten Managements eingeholt und mithilfe von Microsoft Excel ausgewertet.

Allen Frauen, die in dem genannten Zeitraum das ambulante Management nach VBS am Termin in Anspruch genommen hatten, wurde ein Evaluationsbogen ausgehändigt. Dieser sollte anonym und freiwillig innerhalb der ersten zwei Wochen nach der Geburt an die Klinik zurückgesendet werden. Der Fragebogen enthielt vier geschlossene Fragen zu den Hauptthemen: Informationsqualität, Entscheidungsprozess und Sicherheit sowie Wohlbefinden im häuslichen Umfeld.

Zudem hatten die Frauen die Möglichkeit, offenes Feedback und Verbesserungsvorschläge zu geben. Insgesamt wurden 18 ausgefüllte Evaluationsbögen zurückgeschickt. Dies entspricht einer Rücklaufquote von $20,7 \%$.

\section{Ergebnisse/Auswertung}

Anhand der Datenauswertung ist erkennbar, dass auf die Frage, ob der Informations-, Beratungs- und Aufklärungsprozess zur Entscheidungsfindung für das ambulante Management ausreichend war, zwei Frauen eine neutrale Stimme abgaben, während sieben eher zustimmen und neun voll und ganz zustimmten. Die Entscheidung zur Entlassung in das häusliche Umfeld fiel allen Befragten leicht, bis auf eine Frau, die eine neutrale Stimme vergab. Einstimmig ist die Aussage, dass sich die Frauen zu Hause sicher und wohl gefühlt haben, was wiederum auf die Beratungsqualität zurückzuführen ist. Ebenso konsistent war, dass sich alle Frauen erneut für ein ambulantes Procedere entscheiden würden. In Hinblick auf die geringe Anzahl der ausgefüllten Evaluationsbögen ist die Auswertung 
nur bedingt aussagekräftig und kann nicht zur Verallgemeinerung genutzt werden.

\section{Fazit}

Die erhobenen Daten des St. Joseph Krankenhaus BerlinTempelhof beweisen, dass eine ambulante Betreuung nach VBS am Termin eine sichere Alternative zur stationären Versorgung darstellt. Dies gilt sowohl für die Frauen als auch für das Klinikpersonal [10]. Auch die Ressourcenschonung stellt einen wichtigen Aspekt dar.

Hervorzuheben ist, dass durch das ambulante Vorgehen die Physiologie der Geburt gefördert wird und u.a. weniger medizinische Geburtseinleitungen und protrahierte Geburtsverläufe zu beobachten waren. Frauen sollten darin bestärkt werden, selbstbestimmt über den Aufenthaltsort nach VBS zu entscheiden und sich dabei auf klinikinterne und alternative Prozeduren und Überwachungen einzulassen.

Auch wenn weitere umfangreiche und vor allem multizentrische, prospektive Studien [7], für eine bessere Aussagekräftigkeit empfohlen werden, ist die Tendenz statistisch gesichert.

Letztlich basierte dieser Veränderungsprozess auf einer Bachelorarbeit im Studiengang International Midwifery Studies und wurde durch die ärztliche Leitung unterstützt. Wir freuen uns, wenn dieses „kleine Beispiel“ Lust auf mehr macht und wir einen Anstoß für weitere Maßnahmen und Anpassungsprozesse geben können.

\section{Autorinnen / Autoren}

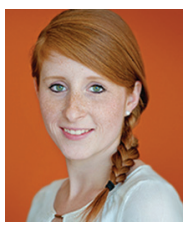

Anne-Katrin Klotzsch ist examinierte Hebamme seit 2014 und akademisiert mit einem Bachelor of Science with First Class Honours in International Midwifery Studies. Neben der Anstellung im Kreißsaal des St. Joseph Krankenhauses Berlin-Tempelhof fungiert sie als Praxisanleiterin für die Studentinnen der Evangelischen Hochschule Berlin im Studiengang Hebammenkunde. Seit 2019 ist sie Mitherausgeberin der Zeitschrift DIE HEBAMME.

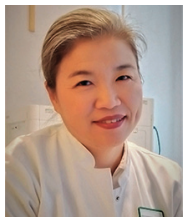

Prof. Dr. Weijing Li ist Ärztin für Frauenheilkunde und Geburtshilfe mit langjähriger Leitungserfahrung in der Geburtsmedizin und Spezialisierung in den Bereichen Pränatalmedizin und Fetalchirurgie. Sie ist als Oberärztin am St. Joseph Krankenhaus Berlin-Tempelhof tätig.

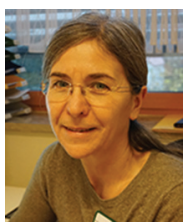

Monique Kaiser ist seit 1995 Hebamme und arbeitete im St. Elisabeth und St. Barbara Krankenhaus in Halle/Saale. Seit 2016 ist sie leitende Hebamme im St. Joseph Krankenhaus Berlin-Tempelhof.
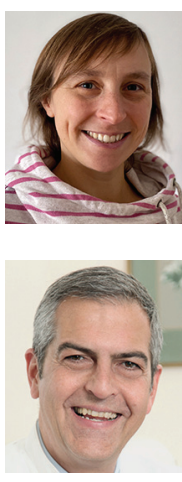

Marie Hurpin ist seit 2010 ausgebildete Hebamme und arbeitete im Kreißsaal des Hôpital Daler-Spital in der Schweiz und in der Maternité des Diaconesses in Paris. Seit 2018 ist sie im St. Joseph Krankenhaus Berlin-Temperhof als Hebamme tätig.

Prof. Dr. med. Michael Abou-Dakn ist Chefarzt der Klinik für Gynäkologie und Geburtshilfe und seit 2019 ärztlicher Direktor im St. Joseph Krankenhaus Berlin-Tempelhof. Zudem ist er Professor für Geburtsmedizin im Fachbereich Hebammenkunde der Evangelischen Hochschule Berlin.

\section{Korrespondenzadresse}

St. Joseph Krankenhaus Berlin-Tempelhof

Klinik für Gynäkologie und Geburtshilfe

Chefarzt Prof. Dr. M. Abou-Dakn

Anne-Katrin Klotzsch

Wüsthoffstr. 15

12101 Berlin

Deutschland

E-Mail: frauenklinik@sjk.de

\section{Literatur}

[1] National Health System (NHS). Greater Manchester and an Eastern Cheshire. Stratetic Clinical Network. Pre-Labour Spontaneous Rupture of Membranes (SROM) at Term (>37 weeks) Guideline. Im Internet: https://www.google.com/url?sa= $t \& r c t=j \& q=\& e s r c=s \&$ source $=$ web\& $c d=\& v e d=2$ ahUKEwjgvMG3OPsAhUBNOwKHYE_D28QFjAAegQIDBAC\&url = https\%3A\% 2F\%2Fwww.england.nhs.uk\%2Fnorth-west\%2Fwp-content\% 2Fuploads\%2Fsites\%2F48\%2F2019\%2F03\%2FGMEC-SROMTerm-37-weeks-plus-guidelines.pdf\&usg = AOvVaw0bLBieK9KJbQs093jLYCMz; Stand: 09/2018

[2] National Health System (NHS). Royal Cornwall Hospitals. NHS Trust. Pre Labour Rupture of Membranes at Term Rupture of Membranes at Term (Term PROM) - Clinical Guideline for management. Im Internet: https://www.google.com/url?sa= $t \& r c t=j \& q=\& e s r c=s \&$ source $=$ web\& $c d=\& v e d=2$ ahUKEwjWkluDJ_sAhVCyaQKHYfMAu0QFjABegQIARAC\&url = https\%3A\%2F\% 2Fdoclibrary-rcht.cornwall.nhs.uk\%2FGET\%2Fd10140814\&usg= AOvVaw3LzLjas91Q-NrGs5dx6o3G: Stand: 10/2020

3] The Royal Australian and New Zealand College of Obstetricians and Gynaecologists (RANZCOG). Term Prelabour Rupture of Membranes (Term PROM). Im Internet: https://www. google.com $/$ url?sa $=$ t\&rct $=j \& q=\& e s r c=s \&$ source $=$ web\&cd=\&ved = 2ahUKEwjNolze-J_sAhUQLewKHf-ADLsQFjAAegQIBBAC\&url = https\%3A\%2F\%2Franzcog.edu.au\% 2FRANZCOG_SITE\%2Fmedia\%2FRANZCOG-MEDIA\%2FWomen $\% 2527$ s\%2520Health\%2FStatement\%2520and\%2520guidelines\%2FClinical-Obstetrics\%2FTerm-Prelabour-Rupture-ofMembranes-(Term-Prom)-(C-Obs-36)-review-2017.pdf\%3Fext \%3D.pdf\&usg = AOvVaw3d2 MAsVcCNDVCdU_pKUAJW: Stand: $03 / 2020$

[4] Deutsche Gesellschaft für Gynäkologie und Geburtshilfe e. V (DGGG), Arbeitsgemeinschaft der Wissenschaftlichen Medizinischen Fachgesellschaften e.V. (AWMF). Empfehlungen zum Vorgehen beim vorzeitigen Blasensprung. Im Internet: https://www.dggg.de/fileadmin/documents/leitlinien/archiviert/federfuehrend/ 
015029_Empfehlungen_zum_Vorgehen_beim_vorzeitigen_Blasensprung/015029_2010.pdf; Stand: 06/2010

[5] Frankhauser C, Bürklin IF, Hodel M et al. Prelabour Rupture of Membranes at Term: In- or outpatient mangement? A survey in birth institutions in the german-speaking part of Switzerland. Z Geburtshilfe Neonatol 2016; 220(5): 207-214

[6] Vetter G, Niklaus A, Hösli I. Ambulantes Management des vorzeitigen Blasensprung (sVBS) am Termin: maternales und perinatales Outcome. Z Geburtshilfe Neonatol 2013; 217-V07_7

[7] Vetter G, Knipprath A, Niklaus A et al. Ambulantes Management bei vorzeitigem Blasensprung (VBS) am Termin. Eine Outcome-Analysestudie bei ambulantem vs. stationärem Management. Z Geburtshilfe Neonatol 2016; 220(5): 215-220

[8] Chacon KM, Bryant Mantha AS, Clapp MA et al. Outpatient expectant management of Premature Rupture of Membranes for women who decline augmentation [14F]. Obstetrics \& Gynecology 2019; 133(1): 65S-66S
[9] Novustat. P-Wert Statistik. Im Internet: https://novustat.com /statistik-glossar/p-wert-statistik.html; Stand: 2020

[10] Klotzsch AK, Abou-Dakn M. Ambulantes Management nach vorzeitigem Blasensprung-eine chancenreiche Alternative. Die Hebamme 2019; 32: 8-13

Bibliografie

Die Hebamme 2021; 34: 50-55

DOI 10.1055/a-1332-8201

ISSN 0932-8122

(C) 2021. Thieme. All rights reserved.

Georg Thieme Verlag KG, Rüdigerstraße 14,

70469 Stuttgart, Germany 